ARTICLE LINK: http://www.sciencedirect.com/science/article/pii/S0191261513000209 PLEASE CITE THIS ARTICLE AS

Han, K., Friesz, T.L., Yao, T., 2013. Existence of simultaneous route and departure choice dynamic user equilibrium. Transportation Research Part B 53, 17-30.

\title{
Existence of Simultaneous Route and Departure Choice Dynamic User Equilibrium
}

\author{
Ke Han ${ }^{\mathrm{a}}$, Terry L. Friesz ${ }^{\mathrm{b}, *}$, Tao Yao ${ }^{\mathrm{b}}$ \\ ${ }^{a}$ Department of Mathematics, Pennsylvania State University, PA 16802, USA. \\ ${ }^{b}$ Department of Industrial and Manufacturing Engineering, Pennsylvania State University, PA 16802, USA.
}

\begin{abstract}
This paper is concerned with the existence of the simultaneous route-and-departure choice dynamic user equilibrium (SRDC-DUE) in continuous time. The SRDC-DUE problem was formulated as an infinite-dimensional variational inequality in Friesz et al. (1993). In deriving our existence result, we employ the generalized Vickrey model (GVM) introduced in Han et al. (2013a.b) to formulate the underlying network loading problem. As we explain, the GVM corresponds to a path delay operator that is provably strongly continuous on the Hilbert space of interest. Finally, we provide the desired SRDC-DUE existence result for general constraints relating path flows to a table of fixed trip volumes without invocation of a priori bounds on the path flows.
\end{abstract}

Keywords:

simultaneous route-and-departure choice dynamic user equilibrium, existence, the generalized Vickrey model, effective delay operator

\section{Introduction}

In this paper we shall consider dynamic traffic assignment (DTA) to be the positive (descriptive) modeling of time-varying flows of automobiles on road networks consistent with established traffic flow theory and travel demand theory. Dynamic User Equilibrium (DUE) is one type of DTA wherein effective unit travel delay for the same purpose is identical for all utilized path and departure time pairs. The relevant notion of travel delay is effective unit travel delay, which is the

\footnotetext{
This work is partially supported by NSF through grant EFRI-1024707, "A theory of complex transportation network design".

${ }^{*}$ Corresponding author

Email addresses: kxh323@psu. edu (Ke Han), tfriesz@psu. edu (Terry L. Friesz), tyy1@engr.psu . edu (Tao Yao)
} 
ARTICLE LINK: http://www.sciencedirect.com/science/article/pii/S0191261513000209 PLEASE CITE THIS ARTICLE AS

Han, K., Friesz, T.L., Yao, T., 2013. Existence of simultaneous route and departure choice dynamic user equilibrium. Transportation Research Part B 53, 17-30.

sum of arrival penalties and actual travel time. For our purposes in this paper, DUE is modeled for the within-day time scale based on fixed travel demands.

In the last two decades there have been many efforts to develop a theoretically sound formulation of dynamic network user equilibrium that is also a canonical form acceptable to scholars and practitioners alike. DUE models tend to be comprised of four essential sub-models:

1. a model of path delay;

2. flow dynamics;

3. flow propagation constraints;

4. a path/departure-time choice model.

Furthermore, analytical DUE models tend to be of two varieties: (1) route choice (RC) user equilibrium (Friesz et al., 1989; Merchant and Nemhauser, 1978a, b; ; Mounce, 2006; Smith and Wisten, 1995; Zhu and Marcotte, 2000); and (2) simultaneous route-and-departure choice (SRDC) dynamic user equilibrium (Friesz et al., 1993, 2001, 2011, 2013; Ran et al., 1996; Wie et al., 2002). For both types of DUE models, the existence of a dynamic user equilibrium in continuous time remains a fundamental issue. A proof of DUE existence is a necessary foundation for qualitative analysis and computational studies. In this paper, we provide a DUE existence result for the SRDC DUE problem when it is formulated as an infinite-dimensional variational inequality of the type presented in Friesz et al. (1993). In this paper, in order to establish a DUE existence result, we study the network loading problem based on the generalized Vickrey model (GVM) proposed in Han et al. (2013a, b). All of our results presented in this paper are more general than any obtained previously for DUE when some version of the point queue model is employed.

\subsection{Formulation of the SRDC user equilibrium}

There are two essential components within the RC or SRDC notions of DUE: (i) the mathematical expression of Nash-like equilibrium conditions, and (ii) a network performance model, which is, in effect, an embedded network loading problem. The embedded network loading problem captures the relationships among arc entry flow, arc exit flow, arc delay and path delay for any path departure rate trajectory.

There are multiple means of expressing the Nash-like notion of a dynamic equilibrium, including the following:

1. a variational inequality (Friesz et al., 1993; Smith and Wisten, 1994, 1995)

2. an evolution equation in an appropriate function space (Mounce, 2006; Smith and Wisten, 1995)

3. a nonlinear complementarity problem (Wie et al., 2002; Han et al., 2011)

4. a differential variational inequality (Friesz et al,, 2001, 2011, 2013; Friesz and Mookherjee, 2006); and

5. a differential complementarity system (Pang et al., 2011).

The variational inequality representation is presently the primary mathematical form employed for both RC and SRDC DUE. The most obvious approach to establishing existence for any of the mathematical representations mentioned above is to convert the problem to an equivalent fixed point problem and then apply Brouwer's fixed point existence theorem. Alternatively, one may use an existence theorem for the particular mathematical representation selected; it should be noted that most such theorems are derived by using Brouwer's famous theorem. So, in effect, all 
ARTICLE LINK: http://www.sciencedirect.com/science/article/pii/S0191261513000209 PLEASE CITE THIS ARTICLE AS

Han, K., Friesz, T.L., Yao, T., 2013. Existence of simultaneous route and departure choice dynamic user equilibrium. Transportation Research Part B 53, 17-30.

proofs of DUE existence employ Brouwer's fixed point theorem, either implicitly or explicitly. One statement of Brouwer's theorem appears as Theorem 2 of Browder (1968). Approaches based on Brouwer's theorem require the set of feasible path flows (departure rates) under consideration to be compact and convex in a Banach space, and typically involve an a priori bound on all the path flows.

We also wish to point out that this paper employs much more general constraints relating path flows to a table of fixed trip volumes than has been previously considered when studying SRDC-DUE. Moreover, in our study of existence, we do not invoke a priori bounds on the path flows to assure boundedness needed for application of Brouwer's theorem. That is, a goal of this paper is to investigate the existence of DUE without making the assumption of $a$ priori bounds for departure rates. Note should be taken of the following fact: the boundedness assumption is less of an issue for the RC DUE by virtue of problem formulation; that is, for RC DUE, the travel demand constraints are of the following form:

$$
\sum_{p \in \mathcal{P}_{i j}} h_{p}(t)=R_{i j}(t) \quad \forall t, \quad \forall(i, j) \in \mathcal{W}
$$

where $\mathcal{W}$ is the set of origin-destination pairs, $\mathcal{P}_{i j}$ is the set of paths connecting $(i, j) \in \mathcal{W}$ and $h_{p}(t)$ is the departure rate along path $p$. Furthermore, $R_{i j}(t)$ represents the rate (not volume) at which travelers leave origin $i$ with the intent of reaching destination $j$ at time $t$; each such trip rate is assumed to be bounded from above. Since 1.11 is imposed pointwise and every path flow $h_{p}$ is nonnegative, we are assured that each $h=\left(h_{p}: p \in \mathcal{P}_{i j},(i, j) \in \mathcal{W}\right)$ are automatically uniformly bounded. On the other hand, the SRDC user equilibrium imposes the following constraints on path flows:

$$
\sum_{p \in \mathcal{P}_{i j}} \int_{t_{0}}^{t_{f}} h_{p}(t) d t=Q_{i j} \quad \forall(i, j) \in \mathcal{W}
$$

where $Q_{i j} \in \mathfrak{R}_{+}^{1}$ is the volume (not rate) of travelers departing node $i$ with the intent of reaching node $j$. The integrals in (1.2) are interpreted as Legesgue; hence, (1.2) alone is not enough to assure bounded path flows. This observation has been the major hurdle to proving existence without the a priori invocation of bounds on path flows. In this paper, we will overcome this difficulty through careful analysis of the GVM and by investigating the effect of user behavior in shaping network flows, in a mathematically intuitive yet rigorous way.

\subsection{Importance of the path delay operator}

Clearly another key component of continuous-time DUE is the path delay operator, typically obtained from dynamic network loading (DNL), which is a subproblem of a complete DUE mode! Any DNL must be consistent with the established path flows and link delay model, and

\footnotetext{
${ }^{1}$ Note that, by referring to the network loading procedure, we are neither employing nor suggesting a sequential approach to the study and computation of DUE. Rather a subset of the equations and inequalites comprising a complete DUE model may be grouped in a way that identifies a traffic assignment subproblem and a network loading subproblem. Such a grouping and choice of names is merely a matter of convenient language that avoids repetitive reference to the same mathematical expressions. Use of such language does not alter the need to solve both the assignment and loading problems consistently and, thus, simultaneously. A careful reading of the mathematical presentation made in subsequent sections makes this quite clear.
} 
ARTICLE LINK: http://www.sciencedirect.com/science/article/pii/S0191261513000209 PLEASE CITE THIS ARTICLE AS

Han, K., Friesz, T.L., Yao, T., 2013. Existence of simultaneous route and departure choice dynamic user equilibrium. Transportation Research Part B 53, 17-30.

DNL is usually performed under the first-in-first-out (FIFO) rule. The properties of the delay operator are critical to proving existence of a solution to the infinite-dimensional variational inequality used to express DUE. In Zhu and Marcotte (2000), using the link delay model introduced by Friesz et al. (1993), the authors showed weak continuity of the path delay operator under the assumption that the path flows are a priori bounded. Their continuity result is superceded by a more general result proven in this paper: the path delay operator of interest is strongly continuous without the assumption of boundedness. Strong continuity without boundedness is central to our proof of existence in the present paper.

In this paper, as a foundation for DNL, we will consider the Vickrey model of congestion first introduced by Vickrey (1969) and later studied by Han et al. (2013a. b). The Vickrey model for a single link is primarily described by an ordinary differential equation (ODE) with discontinuous right hand side. Such irregularity has made it difficult to analyze the Vickery model in continuous time. Fortunately, in this paper, we will be able to take advantage of the closed-form reformulation proposed in Han et al. (2013a b) , then prove the strong continuity of the path delay operator without boundedness of the path flows. This will provide a quite general existence proof for SRDC-DUE based on the generalized Vickrey model.

\subsection{Organization}

The balance of this paper is organized as follows. Section 2 provides essential mathematical background on the concepts that will be used in the paper. Section 3 briefly reviews the formal definition of dynamic user equilibrium and its formulation as a variational inequality. Section 4 4 recaps the generalized Vickrey model (GVM) originally put forward by Han et al. (2013a, b). Section 5 formally discusses the properties of the effective delay operator. The main result of this paper, the existence of an SRDC-DUE when the GVM informs network loading is established in Theorem 5.7 of Section 5.3

\section{Mathematical preliminaries}

A topological vector space is one of the basic structures investigated in functional analysis. Such a space blends a topological structure with the algebraic concept of a vector space. The following is a precise definition.

Definition 2.1. (Topological vector space) A topological vector space $X$ is a vector space over a topological field $\mathbb{F}$ (usually the field of real or complex numbers with their standard topologies) which is endowed with a topology such that vector addition $X \times X \rightarrow X$ and scalar multiplication $\mathbb{F} \times X \rightarrow X$ are continuous functions.

As a consequence of Definition 2.1 all normed vector spaces, and therefore all Banach spaces and Hilbert spaces, are examples of topological vector spaces. Also important is the notion of a seminorm:

Definition 2.2. (Seminorm) A seminorm on a vector space $X$ is a real-valued function $p$ on $X$ such that

(a) $p(x+y) \leq p(x)+p(y)$

(b) $p(\alpha x)=|\alpha| p(x)$

for all $x$ and $y$ in $X$ and all scalars $\alpha$. 
ARTICLE LINK: http://www.sciencedirect.com/science/article/pii/S0191261513000209

\section{PLEASE CITE THIS ARTICLE AS}

Han, K., Friesz, T.L., Yao, T., 2013. Existence of simultaneous route and departure choice dynamic user equilibrium. Transportation Research Part B 53, 17-30.

Definition 2.3. (Locally convex space) A locally convex space is defined to be a vector space $X$ along with a family of seminorms $\left\{p_{i}\right\}_{i \in I}$ on $X$.

As part of our review we make note of the following essential knowledge:

Fact 1. The space of square-integrable real-valued functions on a compact interval $[a, b]$, denoted by $\mathcal{L}^{2}[a, b]$, is a locally convex topological vector space.

Fact 2. The $m$-fold product of the spaces of square-integrable functions $\left(\mathcal{L}^{2}[a, b]\right)^{m}$ is a locally convex topological vector space.

Definition 2.4. (Dual space) The dual space $X^{*}$ of a vector space $X$ is the space of all continuous linear functions on $X$.

Given a vector space $X$, let $\varphi \in X^{*}$ be a continuous linear function on $X$, then we use $\langle\cdot, \cdot\rangle$ to denote the duality pairing of $X$ with its dual space $X^{*}$, that is

$$
\langle\varphi, x\rangle \doteq \varphi(x) \quad \forall x \in X
$$

Another key property we consider without proof is:

Fact 3. The dual space of $\mathcal{L}^{p}[a, b]$ for $1<p<\infty$ has a natural isomorphism with $\mathcal{L}^{q}[a, b]$ where $q$ is such that $1 / p+1 / q=1$. In particular, the dual space of $\mathcal{L}^{2}[a, b]$ is again $\mathcal{L}^{2}[a, b]$.

Let us now give the formal definition of a variational inequality in a topological setting:

Definition 2.5. (Infinite-Dimensional Variational inequality) Let $V$ be a topological vector space and $F: U \rightarrow V^{*}$, where $U \subset V$. The infinite-dimensional variational inequality is posed as the following problem

$$
\left.\begin{array}{c}
\text { find } u^{*} \in U \text { such that } \\
\left\langle F\left(u^{*}\right), u-u^{*}\right\rangle \geq 0 \forall u \in U
\end{array}\right\} V I(F, U)
$$

The key foundation for analysis of existence is the following theorem given in Browder (1968):

Theorem 2.6. Let $K$ be a compact convex subset of the locally convex topological vector space $E, T$ a continuous (single-valued) mapping of $K$ into $E^{*}$. Then there exits $u_{0}$ in $K$ such that

$$
\left\langle T\left(u_{0}\right), u-u_{0}\right\rangle \geq 0
$$

for all $u \in K$.

Proof. See Browder (1968).

Definition 2.7. (Compactness of subspaces) A subset $K$ of a topological space $X$ is called compact if for every arbitrary collection $\left\{U_{\alpha}\right\}_{\alpha \in A}$ of open subsets of $X$ such that

$$
K \subset \bigcup_{\alpha \in A} U_{\alpha}
$$

where $A$ is an arbitrary index set, there is a finite subset I of A such that

$$
K \subset \bigcup_{i \in I} U_{i}
$$


ARTICLE LINK: http://www.sciencedirect.com/science/article/pii/S0191261513000209

\section{PLEASE CITE THIS ARTICLE AS}

Han, K., Friesz, T.L., Yao, T., 2013. Existence of simultaneous route and departure choice dynamic user equilibrium. Transportation Research Part B 53, 17-30.

Definition 2.8. (Sequential compactness) A topological space is sequentially compact if every sequence has a convergent subsequence.

An outgrowth of the concepts and results given above, the following fact is stated without proof:

Fact 4. (Royden and Fitzpatrick, 1988) In metric space (hence topological vector space), the notions of compactness and sequential compactness are equivalent.

The final bit of specialized knowledge about topological vector spaces that we shall need is the following:

Definition 2.9. (Weak convergence in Hilbert space) A sequence of points $\left\{x_{n}\right\}$ in a Hilbert space $\mathcal{H}$ is said to be convergent weakly to a point $x \in \mathcal{H}$, denoted as $x_{n} \rightarrow x$ if

$$
\left\langle x_{n}, y\right\rangle \rightarrow\langle x, y\rangle \quad n \rightarrow \infty
$$

for all $y \in \mathcal{H}$, where $\langle\cdot, \cdot\rangle$ is the inner product on the Hilbert space.

\section{Continuous-time dynamic user equilibrium}

In this section, we will assume the time interval of interest is

$$
\left[t_{0}, t_{f}\right] \subset \mathfrak{R}^{1}
$$

The most crucial component of the DUE model is the path delay operator, which provides the time to traverse any path $p$ per unit of flow departing from the origin of that path. The delay operator is denoted by

$$
D_{p}(t, h) \quad \forall p \in \mathcal{P}
$$

where $\mathcal{P}$ is the set of all paths employed by network users, $t$ denotes the departure time, and $h$ is a vector of departure rates. Throughout the rest of the paper, we stipulate that

$$
h \in\left(\mathcal{L}_{+}^{2}\left[t_{0}, t_{f}\right]\right)^{|\mathcal{P}|}
$$

where $\left(\mathcal{L}_{+}^{2}\left[t_{0}, t_{f}\right]\right)^{|\mathcal{P}|}$ denotes the non-negative cone of the $|\mathcal{P}|$-fold product of the Hilbert space $\mathcal{L}^{2}\left[t_{0}, t_{f}\right]$ of square-integrable functions on the compact interval $\left[t_{0}, t_{f}\right]$. The inner product of the Hilbert space $\left(\mathcal{L}^{2}\left[t_{0}, t_{f}\right]\right)^{|\mathcal{P}|}$ is defined as

$$
\langle u, v\rangle \doteq \int_{t_{0}}^{t_{f}}(u(s))^{T} v(s) d s
$$

where the superscript $T$ denotes transpose of vectors. Moreover, the norm

$$
\|u\|_{\mathcal{L}^{2}} \doteq\langle u, u\rangle^{1 / 2}
$$

is induced by the inner product (3.4).

Next, we need to consider a more general notion of travel cost that will motivate on-time arrivals. To this end, for each $p \in \mathcal{P}$, we introduce the effective unit path delay operator $\Psi_{p}$ : $\left[t_{0}, t_{f}\right] \times\left(\mathcal{L}_{+}^{2}\left[t_{0}, t_{f}\right]\right)^{|\mathcal{P}|} \rightarrow \mathfrak{R}_{++}^{1}$ and define it as follows:

$$
\Psi_{p}(t, h) \doteq D_{p}(t, h)+\mathcal{F}\left(t+D_{p}(t, h)-T_{A}\right)
$$


ARTICLE LINK: http://www.sciencedirect.com/science/article/pii/S0191261513000209

\section{PLEASE CITE THIS ARTICLE AS}

Han, K., Friesz, T.L., Yao, T., 2013. Existence of simultaneous route and departure choice dynamic user equilibrium. Transportation Research Part B 53, 17-30.

where $\mathcal{F}(\cdot)$ is the penalty for early or late arrival relative to the target arrival time $T_{A}$. Note that, for convenience, $T_{A}$ is assumed to be independent of destination. However, that assumption is easy to relax, and the consequent generalization of our model is a trivial extension. We interpret $\Psi_{p}(t, h)$ as the perceived travel cost of driver starting at time $t$ on path $p$ under travel conditions $h$. Presently, our only assumption on such costs is that for each $h \in\left(\mathcal{L}_{+}^{2}\left[t_{0}, t_{f}\right]\right)^{|\mathcal{P}|}$, the vector function $\Psi(\cdot, h):\left[t_{0}, t_{f}\right] \rightarrow \mathfrak{R}_{++}^{|\mathcal{P}|}$ is measurable and strictly positive. The assumption of measurability was used for a measure theory-based argument in Friesz et al. (1993). Later in this paper, we shall discuss other properties of this operator, such as continuity on a Hilbert space. The continuity of effective delay is crucial for applying the general theorems in Browder (1968), especially Theorem 2.6 stated above.

To support the development of a dynamic network user equilibrium model, we introduce some additional constraints. Foremost among these are the flow conservation constraints

$$
\sum_{p \in \mathcal{P}_{i j}} \int_{t_{0}}^{t_{f}} h_{p}(t) d t=Q_{i j} \quad \forall(i, j) \in \mathcal{W}
$$

where $\mathcal{P}_{i j}$ is the set of all paths that connect origin-destination (O-D) pair $(i, j) \in \mathcal{W}$, while $\mathcal{W}$ is the set of all O-D pairs. In addition, $Q_{i j}$ is the fixed travel demand for O-D pair $(i, j)$. Using the notation and concepts we have thus far introduced, the set of feasible solutions for DUE when the effective delay operator $\Psi(\cdot, \cdot)$ is given is

$$
\Lambda=\left\{h \in\left(\mathcal{L}_{+}^{2}\left[t_{0}, t_{f}\right]\right)^{|\mathcal{P}|}: \quad \sum_{p \in \mathcal{P}_{i j}} \int_{t_{0}}^{t_{f}} h_{p}(t) d t=Q_{i j} \quad \forall(i, j) \in \mathcal{W}\right\}
$$

Using a presentation very similar to the above, the notion of a dynamic user equilibrium in continuous time was first introduced by Friesz et al. (1993), who employ a definition tantamount to the following:

Definition 3.1. (Dynamic user equilibrium). A vector of departure rates (path flows) $h^{*} \in \Lambda$ is a dynamic user equilibrium if

$$
h_{p}^{*}(t)>0, p \in \mathcal{P}_{i j} \Longrightarrow \Psi_{p}\left[t, h^{*}(t)\right]=v_{i j} \in \mathfrak{R}_{++}^{1} \quad \forall(i, j) \in \mathcal{W}
$$

We denote the dynamic user equilibrium defined this way by $D U E\left(\Psi, \Lambda,\left[t_{0}, t_{f}\right]\right)$.

In the analysis to follow, we focus on the following infinite-dimensional variational inequality formulation of the DUE problem reported in Theorem 2 of Friesz et al. (1993).

$$
\left.\begin{array}{c}
\text { find } h^{*} \in \Lambda \text { such that } \\
\sum_{p \in \mathcal{P}} \int_{t_{0}}^{t_{f}} \Psi_{p}\left(t, h^{*}\right)\left(h_{p}-h_{p}^{*}\right) d t \geq 0 \\
\forall h \in \Lambda
\end{array}\right\} V I\left(\Psi, \Lambda,\left[t_{0}, t_{f}\right]\right)
$$

The variational inequality formulation $V I\left(\Psi, \Lambda,\left[t_{0}, t_{f}\right]\right)$ expressed above subsumes almost all DUE models regardless of the arc dynamics or the network loading models employed. 
ARTICLE LINK: http://www.sciencedirect.com/science/article/pii/S0191261513000209

$$
\text { PLEASE CITE THIS ARTICLE AS }
$$

Han, K., Friesz, T.L., Yao, T., 2013. Existence of simultaneous route and departure choice dynamic user equilibrium. Transportation Research Part B 53, 17-30.

\section{The dynamic network loading}

A key ingredient of the variational inequality formulation of the DUE (3.10) is the effective delay operator $\Psi(t, \cdot)$, which maps a vector of admissible departure rates to the vector of strictly positive travel costs associated with each route-and-departure-time choice. The problem of predicting time-varying network flows consistent with known travel demands and departure rates (path flows) is usually referred to as the dynamic network loading (DNL) problem. Since effective path delays are constructed from arc delays that depend on arc activity and performance, DNL is intertwined with the determination of effective delay operators.

In this section we present a continuous-time DNL model. This model is based on a reformulation of the Vickrey model (Vickrey, 1969), which we call the generalized Vickrey model $(\mathrm{GVM})$; it was apparently first proposed in Han et al. (2013a b). The generalized Vickrey model determines arc exit flow and the arc traversal time from arc entry flow in an explicit way. This formulation not only leads to a simple and explicit computational scheme, but also makes it easier to conduct rigorous analyses of the arc delay operator and, hence, of the effective path delay operator $\Psi(t, \cdot)$.

\subsection{The generalized Vickrey model}

First introduced in Vickrey (1969), the Vickrey model is based on two key assumptions: (i) vehicles have negligible sizes, and, therefore, any non-empty queue is of negligible size; and (ii) link traversal time consists of a fixed travel time plus a congestion-related arc-traversal delay. Let us introduce the following notations:

$$
\begin{aligned}
u(t): & \text { link entering flow } \\
M: & \text { flow capacity of the bottleneck located at the exit of the link } \\
q(t): & \text { queue size } \\
w(t): & \text { link exit flow } \\
T: & \text { constant free flow travel time } \\
\lambda(t): & \text { link traversal time when the time of entry is } t
\end{aligned}
$$

Then the model is described by the following set of equations.

$$
\begin{gathered}
w(t)= \begin{cases}\min \{u(t-T), M\} & q(t)=0 \\
M & q(t) \neq 0\end{cases} \\
\frac{d q(t)}{d t}=u(t-T)-w(t) \\
\lambda(t)=T+\frac{q(t+T)}{M}
\end{gathered}
$$

Notice that (4.11) and (4.12) amount to an ordinary differential equation (ODE) with a right hand side that is discontinuous in the state variable $q(\cdot)$. Such an ODE has been the main hurdle to further analysis and computation of this model in continuous time. In Han et al. (2013a b) a reformulation of the Vickrey model as a Hamilton-Jacobi equation is proposed and solved with a version of the Lax-Hopf formula (the reader is referred to Evans (2010) for more details on Hamilton-Jacobi equation and Lax-Hopf formula). As a result, the solutions to (4.11)-(4.13) are 
ARTICLE LINK: http://www.sciencedirect.com/science/article/pii/S0191261513000209

$$
\text { PLEASE CITE THIS ARTICLE AS }
$$

Han, K., Friesz, T.L., Yao, T., 2013. Existence of simultaneous route and departure choice dynamic user equilibrium. Transportation Research Part B 53, 17-30.

obtained in closed form. Due to space limitation, we omit further details of relevant analysis, and refer the reader to Han et al. (2013ab).

Let us next introduce the cumulative entering vehicle count $U(\cdot)$ and the exiting vehicle count $\mathcal{W}(\cdot)$ at the entrance and exit of the link of interest, respectively. Furthermore, $U(\cdot)$ is assumed to be non-decreasing and left-continuous. Notice that these latter assumption imply that the link entry flows can be unbounded and possibly contain the dirac-delta function. In contrast, Vickrey's original model requires that the entry flow to be at least Lebesgue integrable. As such, the GVM is more general than the Vickrey model.

Using the notation introduced previously, an equivalent statement of (4.11) through (4.13) is the following:

$$
\begin{aligned}
\mathcal{W}(t) & =\min _{\tau \leq t-T}\{U(\tau)-M \tau\}+M(t-T) \\
q(t) & =U(t-T)-M(t-T)-\min _{\tau \leq t-T}\{U(\tau)-M \tau\} \\
\lambda(t) & =T+\frac{1}{M}\left(U(t)-M(t)-\min _{\tau \leq t}\{U(\tau)-M \tau\}\right)
\end{aligned}
$$

Note that in the system (4.14)-(4.16), all the variables of interest are explicitly stated in terms of the cumulative entering vehicle count $U(\cdot)$. Identities (4.14)-(4.16) will serve as the mathematical formulation of link dynamics in the dynamic network loading sub-problem, as we shall explain shortly. The system (4.14)-(4.16) may also be used for deriving mathematical properties of the effective path delay operator, as is demonstrated in Section 5.1

\subsection{The network model}

It is straightforward to extend the generalized Vickrey model to a network, which is represented as a directed graph $G(N, A)$, where $N$ and $A$ are the set of nodes and arcs, respectively. In order to proceed, we introduce some additional notations. In particular, for each node $v \in N$, let $I^{v}$ be the set of incoming links, $O^{v}$ the set of outgoing links. For each arc $a \in A$, let $u_{a}(t), w_{a}(t)$ be the entry flow and exit flow, respectively. The arc entry/exit flows are the sum of entry/exit flows associated with individual paths using this arc; that is,

$$
u_{a}(t)=\sum_{p \in \mathcal{P}} \delta_{a p} u_{a}^{p}(t), \quad w_{a}(t)=\sum_{p \in \mathcal{P}} \delta_{a p} w_{a}^{p}(t) \quad \forall a \in A
$$

where

$$
\delta_{a p}= \begin{cases}1 & \text { if arc } a \text { belongs to path } p \\ 0 & \text { otherwise }\end{cases}
$$

In equation 4.17, we use $u_{a}^{p}(\cdot)$ to denote the link entering flow associated with path $p$, and $w_{a}^{p}(\cdot)$ to denote the link exiting flow associated with path $p$. Let us also define the cumulative entering vehicle count $U_{a}(t)$ and cumulative exiting vehicle count $W_{a}(t)$, for each $\operatorname{arc} a$. Similarly, each one is disaggregated into quantities associated with each path that uses this arc:

$$
U_{a}(t)=\sum_{p \in \mathcal{P}} \delta_{a p} U_{a}^{p}(t), \quad W_{a}(t)=\sum_{p \in \mathcal{P}} \delta_{a p} W_{a}^{p}(t) \quad \forall a \in A
$$

The arc traversal time function, denoted $\lambda_{a}(\cdot)$, is the time taken to traverse arc $a$ when the time of entry is $t$. The arc exit time function $\tau_{a}(t)$ is defined as $\tau_{a}(t) \doteq t+\lambda_{a}(t)$; that is, $\tau_{a}(t)$ represents the time a car leaves arc $a$ when the time of its entry is $t$. 
ARTICLE LINK: http://www.sciencedirect.com/science/article/pii/S0191261513000209

$$
\text { PLEASE CITE THIS ARTICLE AS }
$$

Han, K., Friesz, T.L., Yao, T., 2013. Existence of simultaneous route and departure choice dynamic user equilibrium. Transportation Research Part B 53, 17-30.

For each group of drivers using the same arc $a$, the ratio of their arrival and departure rates must be the same under first-in-first-out (FIFO). This is expressed as

$$
w_{a}^{p}\left(\tau_{a}(t)\right)= \begin{cases}w_{a}\left(\tau_{a}(t)\right) \cdot \frac{u_{a}^{p}(t)}{u_{a}(t)} & \text { if } u_{a}(t) \neq 0 \\ 0 & \text { if } u_{a}(t)=0\end{cases}
$$

(4.19) uniquely determines the turning percentages at junctions with more than one outgoing links, and is consistent with the FIFO discipline and established route choices. It remains to express the path delay as the sum of finitely many link delays. If we describe path $p \in \mathcal{P}$ as the following sequence of conveniently labeled arcs:

$$
p=\left\{a_{1}, a_{2}, \ldots, a_{i-1}, a_{i}, a_{i+1} \ldots, a_{m(p)}\right\}
$$

where $m(p)$ is number of arcs in path $p$.

It then follows immediately that the arrival time along path $p$, when the departure time at the origin is $t$, can be expressed as a composition of arc exit time functions:

$$
\tau_{p}(t)=\tau_{a_{m(p)}} \circ \ldots \circ \tau_{a_{2}} \circ \tau_{a_{1}}(t) \quad p=\left\{a_{1}, a_{2}, \ldots, a_{m(p)}\right\} \in \mathcal{P}
$$

where the operator $\circ$ means composition, that is, $f \circ g(x) \doteq f(g(x))$.

Now the complete network loading procedure is given by (4.14)-(4.20), which is interpreted as a well defined differential algebraic equation (DAE) system. Moreover, as well shall see in the next section, the (effective) path delay operator defined in this way is strongly continuous from the subset $\Lambda \subset\left(L^{2}\left[t_{0}, t_{f}\right]\right)^{|\mathcal{P}|}$ into $\left(\mathcal{L}^{2}\left[t_{0}, t_{f}\right]\right)^{|\mathcal{P}|}$.

\section{Existence of the DUE}

Existence results for DUE are most general if based on formulation (3.10). Theorem 2.6 for the existence of solutions of variational inequalities in topological spaces can be applied if the operator $\Psi(t, \cdot)$ can be shown to be continuous and the feasible set $\Lambda$ can be shown to be compact. After Section 5.1 addresses the continuity of the effective delay operator, based on the DNL model introduced previously, the last obstacle to proving existence is the compactness of $\Lambda$, which unfortunately does not generally occur in SRDC-DUE. To overcome the aforementioned difficulty, we will consider instead successive finite-dimensional approximations of $\Lambda$, and rely on a topological argument. Such an approach is mathematically rigorous but much more challenging than would be the case if $\Lambda$ were compact in the appropriate Hilbert space. The topological argument and supporting infrastructure for a proof of existence are presented in Section 5.2 and Section 5.3

\subsection{Continuity of the effective path delay operator}

In this section, we will establish continuity of the map $h \mapsto \Psi(\cdot, h)$. These results will be essential for the proof of existence theorem for DUE in Section 5.3. Notice that unlike the argument in Zhu and Marcotte (2000) which requires a priori bound for the path flows, the proof provided here works for unbounded path flows and even distributions, thanks to the generalized Vickrey model.

The next lemma provides a sufficient condition for the continuity of the delay function $\lambda_{a}(\cdot), a \in A$. 
ARTICLE LINK: http://www.sciencedirect.com/science/article/pii/S0191261513000209

\section{PLEASE CITE THIS ARTICLE AS}

Han, K., Friesz, T.L., Yao, T., 2013. Existence of simultaneous route and departure choice dynamic user equilibrium. Transportation Research Part B 53, 17-30.

Lemma 5.1. Consider an arc $a \in A$, with inflow $u_{a}(\cdot)$. Under the generalized Vickrey model expressed in (4.14)-(4.16), the arc delay function $\lambda_{a}(\cdot)$ is continuous if $u_{a}(\cdot) \in \mathcal{L}^{2}\left[t_{0}, t_{f}\right]$.

Proof. Assume that $u_{a}(\cdot) \in \mathcal{L}^{2}\left[t_{0}, t_{f}\right]$, then $u_{a}(\cdot) \in \mathcal{L}^{1}\left[t_{0}, t_{f}\right]$. Therefore the cumulative entering vehicle count

$$
U_{a}(t) \doteq \int_{t_{0}}^{t} u_{a}(s) d s
$$

is absolutely continuous. It is straightforward to verify that the following quantity is continuous.

$$
q_{a}(t) \doteq U_{a}(t)-M_{a} t-\min _{\tau \leq t-T_{a}}\left\{U_{a}(\tau)-M_{a} \tau\right\}
$$

where $q_{a}(t)$ denotes the queue length, $M_{a}$ denotes the bottleneck capacity and $T_{a}$ denotes the constant free flow time. By (4.16), the function $\lambda_{a}(\cdot)$ is continuous.

The next lemma is a technical result that will facilitate the proof of Theorem 5.3

Lemma 5.2. Let $g_{n}(\cdot):\left[a_{1}, b_{1}\right] \rightarrow\left[a_{2}, b_{2}\right], n \geq 1$ be a sequence of functions such that $g_{n}$ converges to $g(\cdot):\left[a_{1}, b_{1}\right] \rightarrow\left[a_{2}, b_{2}\right]$ uniformly. In addition, assume $f(\cdot):\left[a_{2}, b_{2}\right] \rightarrow \mathfrak{R}^{1}$ is continuous. Then the following uniform convergence holds.

$$
f\left(g_{n}(\cdot)\right) \longrightarrow f(g(\cdot)) \quad n \longrightarrow \infty
$$

Proof. According to the Heine-Cantor theorem (Royden and Fitzpatrick, 1988), $f(\cdot)$ is uniformly continuous on $\left[a_{2}, b_{2}\right]$. It follows that, for every $\varepsilon>0$, there exists $\delta>0$ such that for any $y_{1}, y_{2} \in\left[a_{2}, b_{2}\right]$, whenever $\left|y_{1}-y_{2}\right|<\delta$, the inequality

$$
\left|f\left(y_{1}\right)-f\left(y_{2}\right)\right| \leq \varepsilon
$$

holds. Moreover, by the uniform convergence of $g_{n}$, there exists some $N>0$ such that, for all $n>N$, we have

$$
\left|g_{n}(x)-g(x)\right|<\delta \quad \forall x \in\left[a_{1}, b_{1}\right]
$$

Thus, for every $n>N$,

$$
\left|f\left(g_{n}(x)\right)-f(g(x))\right| \leq \varepsilon \quad \forall x \in\left[a_{1}, b_{1}\right]
$$

Theorem 5.3. Under the network loading model described in Section 4 the effective path delay operator $\Psi(t, \cdot): \Lambda \rightarrow\left(\mathcal{L}^{2}\left[t_{0}, t_{f}\right]\right)^{|\mathcal{P}|}, h \mapsto \Psi(\cdot, h)$ is well-defined and continuous.

Proof. For each $h \in \Lambda$, the functions $\Psi_{p}(\cdot, h), p \in \mathcal{P}$ are uniquely determined by the network loading procedure. To show that the effective path delay operator is well-defined, it remains to show that $\Psi(\cdot, h) \in\left(\mathcal{L}^{2}\left[t_{0}, t_{f}\right]\right)^{|\mathcal{P}|}$ for each $h \in \Lambda$. Notice that there exists an upper bound for the path delays regardless of the network flow profile:

$$
D_{p}(t, h) \leq \sum_{a \in p}\left\{\frac{1}{M_{a}} \sum_{(i, j) \in \mathcal{W}} Q_{i j}+T_{a}\right\} \quad \forall h \in \Lambda, \forall p \in \mathcal{P}, \forall t \in\left[t_{0}, t_{f}\right]
$$


ARTICLE LINK: http://www.sciencedirect.com/science/article/pii/S0191261513000209

\section{PLEASE CITE THIS ARTICLE AS}

Han, K., Friesz, T.L., Yao, T., 2013. Existence of simultaneous route and departure choice dynamic user equilibrium. Transportation Research Part B 53, 17-30.

where $M_{a}, T_{a}$ are the bottleneck capacity and the free flow time respectively, that are associated with arc $a$. Recall the definition of the effective path delay (3.6):

$$
\Psi_{p}(t, h)=D_{p}(t, h)+\mathcal{F}\left(t+D_{p}(t, h)-T_{A}\right)
$$

Since $\mathcal{F}(\cdot)$ is continuous, the uniform boundedness of $D_{p}(t, h)$, as shown in 5.21 , thus implies the uniform boundedness of $\Psi_{p}(t, h)$ for all $h \in \Lambda, p \in \mathcal{P}$ and $t \in\left[t_{0}, t_{f}\right]$. This leads to the conclusion that $\Psi(\cdot, h) \in\left(\mathcal{L}^{2}\left[t_{0}, t_{f}\right]\right)^{|\mathcal{P}|}$ for all $h \in \Lambda$.

With the preceding as background, the proof of continuity of the effective delay operator may be given in five parts.

Part 1. We first focus on a single link $a$. For the convenience of notations, the subscript $a$ will be dropped for now. Consider a sequence of entering flows $u_{v}, v \geq 1$ that converge to $u$ in the $\mathcal{L}^{2}$-norm, that is,

$$
\left\|u_{v}-u\right\|_{2} \doteq\left(\int_{t_{0}}^{t_{f}}\left(u_{v}(t)-u(t)\right)^{2} d t\right)^{1 / 2} \longrightarrow 0 \text { as } v \longrightarrow \infty
$$

Consider the cumulative entering vehicle counts

$$
\left\{\begin{array}{l}
U_{v}(t) \doteq \int_{t_{0}}^{t} u_{v}(s) d s \quad v \geq 1 \\
U(t) \doteq \int_{t_{0}}^{t} u(s) d s
\end{array} \quad t \in\left[t_{0}, t_{f}\right]\right.
$$

Then $U_{v}$ converge to $U$ uniformly on $\left[t_{0}, t_{f}\right]$ : this is due to the following simple observation

$$
\left|U_{v}(t)-U(t)\right| \leq \int_{t_{0}}^{t}\left|u_{v}(s)-u(s)\right| d s \leq\left\|u_{v}-u\right\|_{1} \leq\left(t_{0}-t_{f}\right)^{1 / 2}\left\|u_{v}-u\right\|_{2} \longrightarrow 0
$$

where $\|\cdot\|_{1}$ is the norm in $\mathcal{L}^{1}\left[t_{0}, t_{f}\right]$. The last inequality of 5.22 is a version of Jenssen's inequality.

Part 2. Define $R(\tau) \doteq U(\tau)-M \tau, R_{v}(\tau) \doteq U_{v}(\tau)-M \tau, \tau \in\left[t_{0}, t_{f}\right]$, where $M$ is the bottleneck capacity. We claim the following uniform convergence:

$$
\min _{\tau \leq t}\left\{R_{v}(\tau)\right\} \rightarrow \min _{\tau \leq t}\{R(\tau)\} \quad \forall t \in\left[t_{0}, t_{f}\right]
$$

Indeed, for any $\varepsilon>0$, by the uniform convergence of $U_{v}, v \geq 1$, we can choose $N$ such that for all $v \geq N$, the following inequality holds

$$
\left|U_{v}(t)-U(t)\right| \leq \varepsilon \quad \forall t \in\left[t_{0}, t_{f}\right]
$$

Fix any $t$, if $v \geq N$, then

$$
\left|R_{\nu}(\tau)-R(\tau)\right|=\left|U_{\nu}(\tau)-U(\tau)\right| \leq \varepsilon \quad \forall \tau \in\left[t_{0}, t_{f}\right]
$$

Define $\hat{\tau}=\operatorname{argmin}_{\tau \leq t}\{R(\tau)\}$. By (5.24) we have

$$
\min _{\tau \leq t}\left\{R_{\nu}(\tau)\right\} \leq R_{\nu}(\hat{\tau}) \leq R(\hat{\tau})+\varepsilon=\min _{\tau \leq t}\{R(\tau)\}+\varepsilon
$$


ARTICLE LINK: http://www.sciencedirect.com/science/article/pii/S0191261513000209

\section{PLEASE CITE THIS ARTICLE AS}

Han, K., Friesz, T.L., Yao, T., 2013. Existence of simultaneous route and departure choice dynamic user equilibrium. Transportation Research Part B 53, 17-30.

On the other hand, define $\hat{\tau}_{v}=\operatorname{argmin}_{\tau \leq t}\left\{R_{v}(\tau)\right\}$ for each $v \geq 1$. Then given $v \geq N$, it must hold that

$$
\min _{\tau \leq t}\{R(\tau)\} \leq R\left(\hat{\tau}_{v}\right) \leq R^{(v)}\left(\hat{\tau}_{v}\right)+\varepsilon=\min _{\tau \leq t}\left\{R_{v}(\tau)\right\}+\varepsilon
$$

Taken together, 5.25) and 5.26 imply

$$
\left|\min _{\tau \leq t}\left\{R_{v}\right\}-\min _{\tau \leq t}\{R(\tau)\}\right| \leq \varepsilon \quad \forall v \geq N
$$

Since $t$ is arbitrary, the claim is demonstrated.

Part 3. An immediate consequence of Part 2 and (4.14)-(4.16) is the following uniform convergence

$$
W_{v}(t) \longrightarrow W(t), \quad q_{v}(t) \longrightarrow q(t), \quad \lambda_{v}(t) \longrightarrow \lambda(t), \quad \tau_{v}(t) \longrightarrow \tau(t) \quad v \longrightarrow \infty
$$

for which we employ notation whose meaning is transparent. The next step is to extend such convergence to the whole network. Consider the sequence of departure rates $h_{v}$ converging to $h$ in the $\|\cdot\|_{\mathcal{L}^{2}}$ norm. By the definition (3.5), this implies each path flow $h_{p, v}(\cdot) \rightarrow h_{p}$ in the $\|\cdot\|_{2}$ norm, for all $p \in \mathcal{P}$. A simple induction based on results established in Part 2 yields, as $v \longrightarrow \infty$,

$$
U_{a, v}(t) \longrightarrow U_{a}(t), \quad W_{a, v}(t) \longrightarrow W_{a}(t), \quad D_{a, v}(t) \longrightarrow D_{a}(t), \quad \tau_{a, v}(t) \longrightarrow \tau_{a}(t)
$$

uniformly for all $a \in A$.

Part 4. We will show next the uniform convergence of the path delay function $D_{p}\left(\cdot, h_{v}\right) \rightarrow$ $D_{p}(\cdot, h)$, based on 5.28). Recall the path exit time function 4.20.

$$
\tau_{p}(t)=\tau_{a_{m(p)}} \circ \ldots \circ \tau_{a_{2}} \circ \tau_{a_{1}}(t) \quad p=\left\{a_{1}, a_{2}, \ldots, a_{m(p)}\right\} \in \mathcal{P}
$$

We start by showing that $\tau_{a_{2}, v} \circ \tau_{a_{1}, v}(t) \rightarrow \tau_{a_{2}} \circ \tau_{a_{1}}(t)$ uniformly.

For every $v \geq 1$, since the inflow of arc $a_{2}$ is square-integrable, $\tau_{a_{2}, v}(\cdot)$ is continuous by Lemma 5.1 This means that $\tau_{a_{2}}(\cdot)$ is also continuous since it is the uniform limit of $\tau_{a_{2}, v}(\cdot)$. Lemma 5.2 then implies that $\tau_{a_{2}}\left(\tau_{a_{1}, v}(\cdot)\right)$ converges uniformly to $\tau_{a_{2}}\left(\tau_{a_{1}}(\cdot)\right)$, that is, for any $\varepsilon>0$, there exists an $N_{1}>0$ such that for all $v>N_{1}$,

$$
\left|\tau_{a_{2}}\left(\tau_{a_{1}, v}(t)\right)-\tau_{a_{2}}\left(\tau_{a_{1}}(t)\right)\right|<\varepsilon / 2 \quad \forall t \in\left[t_{0}, t_{f}\right]
$$

Moreover, there exists some $N_{2}>0$ such that for all $v>N_{2}$,

$$
\left|\tau_{a_{2}, v}(t)-\tau_{a_{2}}(t)\right|<\varepsilon / 2 \quad \forall t \in\left[t_{0}, t_{f}\right]
$$

Now let $N_{0}=\max \left\{N_{1}, N_{2}\right\}$. Then for any $v>N_{0}$ and any $t \in\left[t_{0}, t_{f}\right]$,

$$
\begin{aligned}
& \left|\tau_{a_{2}, v}\left(\tau_{a_{1}, v}(t)\right)-\tau_{a_{2}}\left(\tau_{a_{1}}(t)\right)\right| \\
\leq & \left|\tau_{a_{2}, v}\left(\tau_{a_{1}, v}(t)\right)-\tau_{a_{2}}\left(\tau_{a_{1}, v}(t)\right)\right|+\left|\tau_{a_{2}}\left(\tau_{a_{1}, v}(t)\right)-\tau_{a_{2}}\left(\tau_{a_{1}}(t)\right)\right| \\
< & \varepsilon / 2+\varepsilon / 2=\varepsilon
\end{aligned}
$$

This shows the desired uniform convergence $\tau_{a_{2}, v} \circ \tau_{a_{1}, v}(t) \rightarrow \tau_{a_{2}} \circ \tau_{a_{1}}(t)$.

The uniform convergence $\tau_{p, v}(\cdot) \rightarrow \tau_{p}(\cdot)$ follows immediately by (5.29) and mathematical induction with Lemma 5.2. As a result, we obtain the uniform convergence of path delay

$$
D_{p}\left(\cdot, h_{v}\right) \longrightarrow \underset{13}{D_{p}(\cdot, h) \quad v \longrightarrow \infty}
$$


ARTICLE LINK: http://www.sciencedirect.com/science/article/pii/S0191261513000209

$$
\text { PLEASE CITE THIS ARTICLE AS }
$$

Han, K., Friesz, T.L., Yao, T., 2013. Existence of simultaneous route and departure choice dynamic user equilibrium. Transportation Research Part B 53, 17-30.

Part 5. Finally, recall the definition of the effective delay

$$
\Psi(t, h)=D_{p}(t, h)+\mathcal{F}\left(t+D_{p}(t, h)-T_{A}\right)
$$

Note that $\mathcal{F}(\cdot)$ is continuous, the following uniform convergence follows by Lemma 5.2

$$
\mathcal{F}\left(t+D_{p}\left(t, h_{v}\right)-T_{A}\right) \longrightarrow \mathcal{F}\left(t+D_{p}(t, h)-T_{A}\right) \quad v \longrightarrow \infty
$$

We conclude that the effective delay $\Psi_{p}\left(\cdot, h_{v}\right)$ converges uniformly to $\Psi_{p}(\cdot, h)$. The desired convergence in the $\|\cdot\|_{\mathcal{L}^{2}}$ norm now follows since the interval $\left[t_{0}, t_{f}\right]$ is compact.

\subsection{Alternative definition of effective path delay}

The integrals employed in defining the feasible domain $(3.8)$ are not enough to assure bounded path flows $h_{p}, p \in \mathcal{P}$. This observation is the fundamental hurdle to providing existence of the DUE solution. One of the main accomplishments of this paper is to address the boundedness of path flows not only for the proof of existence result but also for future analysis and estimation of network flows. In this section, we will present an alternative formulation of the effective path delay $\Psi_{p}(t, h)$, where that alternative formulation will facilitate our analysis leading to the proof of our main result, Theorem5.6

As a motivation, let us recall the effective delay operator

$$
\Psi_{p}(t, h) \doteq D_{p}(t, h)+\mathcal{F}\left(t+D_{p}(t, h)-T_{A}\right)
$$

In order to simplify our analysis, it is convenient to rewrite 5.30 in a slightly different form. In particular, for each O-D pair $(i, j) \in \mathcal{W}$, let us introduce the cost function $\phi_{i j}(\cdot):\left[t_{0}, t_{f}\right] \rightarrow \mathfrak{R}_{+}^{1}$, which is a function of departure time, and $\psi_{i j}(\cdot):\left[t_{0}, t_{f}\right] \rightarrow \mathfrak{R}_{+}^{1}$, which is a function of arrival time. As we shall explain below, the users' travel costs can be alternatively expressed using functions $\phi_{i j}(\cdot)$ and $\psi_{i j}(\cdot)$. Given any origin-destination pair $(i, j) \in \mathcal{W}$, and any driver who departs from the origin at time $t_{d}$, and arrives at destination at $t_{a}$, his/her travel cost is expressed as $\phi_{i j}\left(t_{d}\right)+\psi_{i j}\left(t_{a}\right)$.

Fix any vector of path flows $h \in \Lambda$, recall the path exit time function $\tau_{p}(t)=t+D_{p}(t, h)$ where $t$ denotes departure time. Then (5.30) can be equivalently written as

$$
\Psi_{p}(t, h)=-t+\tau_{p}(t)+\mathcal{F}\left(\tau_{p}(t)-T_{A}\right)=\phi_{i j}(t)+\psi_{i j}\left(\tau_{p}(t, h)\right)
$$

where

$$
\phi_{i j}(t) \doteq-t, \quad \psi_{i j}\left(\tau_{p}(t)\right) \doteq \tau_{p}(t)+\mathcal{F}\left(\tau_{p}(t)-T_{A}\right)
$$

Remark 5.4. In Bressan and Han (2011, 2012, 2013), the unit travel cost is measured in terms of $\phi_{i j}(\cdot)$ and $\psi_{i j}(\cdot)$. In other words, the general effective delay (5.30) can be alternatively evaluated as a sum of costs at the beginning and at the end of each driver's trip.

In Section 5.3 that follows, we will exploit this alternative representative of effective path delay (cost) to establish existence. To prepare for the existence proof, we consider a general network $G(N, A)$, and associate to each O-D pair $(i, j)$ the pair of cost functions $\phi_{i j}(\cdot)$ and $\psi_{i j}(\cdot)$. We make the following two assumptions regarding $\phi_{i j}(\cdot)$ and $\psi_{i j}(\cdot)$ and the underlying link performance model. 
ARTICLE LINK: http://www.sciencedirect.com/science/article/pii/S0191261513000209

\section{PLEASE CITE THIS ARTICLE AS}

Han, K., Friesz, T.L., Yao, T., 2013. Existence of simultaneous route and departure choice dynamic user equilibrium. Transportation Research Part B 53, 17-30.

A1. For each $(i, j) \in \mathcal{W}, \phi_{i j}(\cdot)$ and $\psi_{i j}(\cdot)$ are continuous on $\left[t_{0}, t_{f}\right]$. Moreover, $\phi_{i j}(\cdot)$ is monotonically decreasing while $\psi_{i j}(\cdot)$ is monotonically increasing. In addition, we assume that $\phi_{i j}(\cdot)$ is Lipschitz continuous with constant $L_{i j}$; and there exists $\Delta_{i j}>0$ such that

$$
\psi_{i j}\left(t_{2}\right)-\psi_{i j}\left(t_{1}\right) \geq \Delta_{i j}\left(t_{2}-t_{1}\right) \quad \forall t_{0} \leq t_{1}<t_{2} \leq t_{f}
$$

A2. Each link $a \in A$ of the network has a finite exit flow capacity $M_{a}<\infty$.

Inequality 5.33 requires that the arrival cost function $\psi_{i j}(\cdot)$ is strictly increasing, and the rate of increase is bounded below by $\Delta_{i j}$. In the case where $\psi_{i j}(\cdot)$ is continuously differentiable, this assumption is equivalent to requiring that $\frac{d}{d t} \psi_{i j}(t) \geq \Delta_{i j}>0 t \in\left[t_{0}, t_{f}\right]$, which is further equivalent to $\frac{d}{d t} \psi_{i j}(t)>0 t \in\left[t_{0}, t_{f}\right]$, due to compactness. As a special case, given the effective delay of the form (5.31) and (5.32), $\mathbf{A 1}$ reduces to the following assumptions.

A1'. $\mathcal{F}(\cdot)$ is continuous on $\left[t_{0}, t_{f}\right]$ and satisfies

$$
\mathcal{F}\left(t_{2}\right)-\mathcal{F}\left(t_{1}\right) \geq \Delta\left(t_{2}-t_{1}\right) \quad \forall t_{0} \leq t_{1}<t_{2} \leq t_{f}
$$

for some $\Delta>-1$.

Assumption $\mathbf{A 2}$ applies to all link dynamics that impose an exit flow capacity; examples include the Vickrey model (Vickrev, 1969) and the Lighthill-Whitham-Richards model (Lighthill and Whitham, 1955; Richards, 1956; Han et al., 2012).

Remark 5.5. Our proposed formulation of travel cost subsumes another well-known class of travel cost functions that are employed in, e.g. Pang et al. (2011) and Yao et al. (2010). Namely, given positive constants $\alpha, \beta$ and $\gamma$, the travel cost of road user is expressed as

$$
\alpha\left(t_{a}-t_{d}\right)+ \begin{cases}\beta\left(T-t_{a}\right) & t_{a} \leq T_{A} \\ \gamma\left(t_{a}-T\right) & t_{a}>T_{A}\end{cases}
$$

where $t_{d}, t_{a}$ denote departure and arrival times, respectively. $T_{A}$ is the desired arrival time. It is assumed that $\gamma>\alpha>\beta$. The expression in (5.34) can be rewritten as

$$
\begin{aligned}
\alpha\left(t_{a}-t_{d}\right)+ \begin{cases}\beta\left(T_{A}-t_{a}\right) & t_{a} \leq T_{A} \\
\gamma\left(t_{a}-T_{A}\right) & t_{a}>T_{A}\end{cases} & =-\alpha t_{d}+ \begin{cases}(\alpha-\beta) t_{a}+\beta T_{A} & t_{a} \leq T_{A} \\
(\alpha+\gamma) t_{a}-\gamma T_{A} & t_{a}>T_{A}\end{cases} \\
& =\phi\left(t_{d}\right)+\psi\left(t_{a}\right)
\end{aligned}
$$

where

$$
\phi\left(t_{d}\right) \doteq-\alpha t_{d}, \quad \psi\left(t_{a}\right) \doteq \begin{cases}(\alpha-\beta) t_{a}+\beta T_{A} & t_{a} \leq T_{A} \\ (\alpha+\gamma) t_{a}-\gamma T_{A} & t_{a}>T_{A}\end{cases}
$$

One can easily check that such $\phi(\cdot)$ and $\psi(\cdot)$ satisfy assumption $\mathbf{A 1}$.

In view of the preceding assumptions $\mathbf{A 1}$ and $\mathbf{A 2}$, we are prompted to define the following:

$$
\begin{aligned}
\phi_{\max }^{\prime} & \doteq \max _{(i, j) \in \mathcal{W}} L_{i j}>0 \\
\psi_{\min }^{\prime} & \doteq \min _{(i, j) \in \mathcal{W}} \Delta_{i j}>0 \\
M^{\max } & \doteq \max _{a \in A} M_{a}<+\infty
\end{aligned}
$$


ARTICLE LINK: http://www.sciencedirect.com/science/article/pii/S0191261513000209

\section{PLEASE CITE THIS ARTICLE AS}

Han, K., Friesz, T.L., Yao, T., 2013. Existence of simultaneous route and departure choice dynamic user equilibrium. Transportation Research Part B 53, 17-30.

\subsection{Existence of solution to the variational inequality}

The classical result explained by Theorem 2.6 will be the key ingredient for the proof of existence of the DUE solution. Using the same notation as in Theorem 2.6, the underlying topological vector space $E$ will be instantiated by $\left(\mathcal{L}^{2}\left[t_{0}, t_{f}\right]\right)^{|\mathcal{P}|}$, which is a locally convex topological vector space. The dual space $E^{*}$ will be again $\left(\mathcal{L}^{2}\left[t_{0}, t_{f}\right]\right)^{|\mathcal{P}|}$.

Theorem 5.6. (Existence of DUE) Let assumptions $\mathbf{A 1}$ and $\mathbf{A 2}$ hold. In addition, assume that the effective delay operator $\Psi: \Lambda \rightarrow\left(\mathcal{L}^{2}\left[t_{0}, t_{f}\right]\right)^{|\mathcal{P}|}$ is continuous. Then the dynamic user equilibrium problem as in Definition 3.1 has a solution.

Proof. The proof is divided into four parts.

Part 1. Our strategy for demonstrating existence is to adapt Theorem 2.6 to the locally convex topological vector space $\left(\mathcal{L}^{2}\left[t_{0}, t_{f}\right]\right)^{|\mathcal{P}|}$, and its subset $\Lambda$. By assumption, the map $h \mapsto \Psi(\cdot, h)$ is continuous from $\Lambda$ to the space of $\left(\mathcal{L}^{2}\left[t_{0}, t_{f}\right]\right)^{|\mathcal{P}|}$. If $\Lambda$ were compact and convex, we would immediately demonstrate the desired result. However, $\Lambda$ is bounded, closed and convex, but not compact in $\left(\mathcal{L}^{2}\left[t_{0}, t_{f}\right]\right)^{|\mathcal{P}|}$.

Part 2. We will instead employ finite-dimensional approximations of $\Lambda$. In order to proceed, consider for each $n \geq 1$ the uniform partition of interval $\left[t_{0}, t_{f}\right]$ with $2^{n}$ sub-intervals 2

$$
\begin{aligned}
t_{0} & =t^{0}<t^{1}<t^{2} \ldots<t^{2^{n}}=t_{f} \\
t^{i}-t^{i-1} & =\frac{t_{f}-t_{0}}{2^{n}} \quad i=1, \ldots, 2^{n}
\end{aligned}
$$

Then consider the following sequence of finite-dimensional subsets

$$
\Lambda_{n} \doteq\left\{h \in \Lambda: \quad h_{p}(\cdot) \text { is constant on }\left[t^{i-1}, t^{i}\right) \quad p \in \mathcal{P}\right\} \subset \Lambda \quad n \geq 1
$$

We claim that for each $n \geq 1, \Lambda_{n}$ is compact and convex in $\left(\mathcal{L}^{2}\left[t_{0}, t_{f}\right]\right)^{|\mathcal{P}|}$. Indeed, given any $h^{n, 1}, h^{n, 2} \in \Lambda_{n}$, and $\alpha \in[0,1], \alpha h^{n, 1}+(1-\alpha) h^{n, 2}$ is clearly nonnegative and constant on each $\left[t^{i-1}, t^{i}\right]$ for $i=1, \ldots, 2^{n}$. In addition, for any origin-destination pair $(i, j) \in \mathcal{W}$, by definition (3.8),

$$
\sum_{p \in \mathcal{P}_{i j}} \int_{t_{0}}^{t_{f}} \alpha h^{n, 1}(t)+(1-\alpha) h^{n, 2}(t) d t=\alpha Q_{i j}+(1-\alpha) Q_{i j}=Q_{i j}
$$

This verifies that $\Lambda_{n}$ is convex. To see the compactness of $\Lambda_{n}$, we define the map $\mu: \Lambda_{n} \rightarrow$ $\mathfrak{R}_{+}^{2^{n} \times|\mathcal{P}|}, h \mapsto\left(a_{i, p}: 1 \leq i \leq 2^{n}, p \in \mathcal{P}\right)$ where each vector $\left(a_{1, p}, \ldots, a_{2^{n}, p}\right)$ is the coordinate of $h_{p}$ under the natural basis $\left\{e_{n}^{i}\right\}_{i=1}^{n}$, where

$$
e_{n}^{i}(t)= \begin{cases}1 & t \in\left[t^{i-1}, t^{i}\right) \\ 0 & \text { else }\end{cases}
$$

Clear, the map $\mu$ is one-to-one. Now fix $n$ and consider an arbitrary sequence $\left\{h^{n, v}\right\}_{v \geq 1} \subset \Lambda_{n}$, as well as the sequence of their images $\left\{\mu\left(h^{n, v}\right)\right\}_{v \geq 1} \subset \mathfrak{R}_{+}^{2^{n} \times|\mathcal{P}|}$. By $[3.8)$, the latter sequence is uniformly bounded by the following quantity

$$
\max _{(i, j) \in \mathcal{W}} \frac{2^{n} Q_{i j}}{t_{f}-t_{0}}
$$

\footnotetext{
${ }^{2}$ The choice of the number of sub-intervals is quite flexible: as long as the size of the sub-intervals approaches zero as $n \rightarrow+\infty$, our framework for showing existence will work.
} 
ARTICLE LINK: http://www.sciencedirect.com/science/article/pii/S0191261513000209

\section{PLEASE CITE THIS ARTICLE AS}

Han, K., Friesz, T.L., Yao, T., 2013. Existence of simultaneous route and departure choice dynamic user equilibrium. Transportation Research Part B 53, 17-30.

According to the Bolzano-Weierstrass theorem, there exists a convergent subsequence $\left\{\mu\left(h^{n, v^{\prime}}\right)\right\}_{v^{\prime} \geq 1}$ with limit $y^{*} \in \mathfrak{R}_{+}^{2^{n} \times|\mathcal{P}|}$. By construction, the corresponding subsequence $\left\{h^{\left.n, v^{\prime}\right)}\right\}_{v^{\prime} \geq 1}$ must converge uniformly to $\hat{h}^{n} \doteq \mu^{-1}\left(y^{*}\right)$. In view of the compact interval $\left[t_{0}, t_{f}\right]$, we conclude that this convergence holds also in the $\|\cdot\|_{\mathcal{L}^{2}}$ norm. It now remains to show $\hat{h}^{n} \in \Lambda_{n}$. Clearly $\hat{h}^{n} \geq 0$ and is constant on the sub-intervals $\left[t^{i-1}, t^{i}\right), i=1, \ldots, 2^{n}$. Moreover, since $h^{n, v^{\prime}}$ are uniformly bounded, by the Dominated Convergence Theorem,

$$
Q_{i j}=\lim _{v^{\prime} \rightarrow \infty} \sum_{p \in \mathcal{P}_{i j}} \int_{t_{0}}^{t_{f}} h_{p}^{n, v^{\prime}}(t) d t=\sum_{p \in \mathcal{P}_{i j}} \int_{t_{0}}^{t_{f}} \hat{h}_{p}^{n}(t) d t \quad \forall(i, j) \in \mathcal{W}
$$

This implies $\hat{h}^{n} \in \Lambda_{n}$. Thereby the sequential compactness, and hence the compactness, of $\Lambda_{n}$ is substantiated.

Part 3. For each $n \geq 1$, apply Theorem 2.6 to $\Lambda_{n}$ and obtain $h^{n, *} \in \Lambda_{n}$ such that

$$
\left\langle\Psi\left(\cdot, h^{n, *}\right), h^{n}(\cdot)-h^{n, *}(\cdot)\right\rangle \geq 0 \quad \forall h^{n} \in \Lambda_{n}
$$

Where the $\langle$,$\rangle is the inner product (duality) defined in (3.4). Inequality (5.39) implies that given$ any $p \in \mathcal{P}$, if $h_{p}^{n, *}(t)>0, t \in\left[t^{j}, t^{j+1}\right)$, then the following is true.

$$
\int_{t^{j}}^{t^{j+1}} \Psi_{p}\left(t, h^{n, *}\right) d t=\min _{0 \leq k \leq 2^{n}} \int_{t^{k}}^{t^{k+1}} \Psi_{p}\left(t, h^{n, *}\right) d t
$$

In view of 5.35), 5.36) and 5.37, we choose any constant $\mathcal{M}$ such that

$$
\mathcal{M}>\frac{3 M^{\max } \phi_{\max }^{\prime}}{\psi_{\min }^{\prime}}
$$

We then claim that $h_{p}^{n, *}(t) \leq \mathcal{M}, \forall t \in\left[t_{0}, t_{f}\right], \forall p \in \mathcal{P}, \forall n \geq 1$. Otherwise, assume there exists some $p \in \mathcal{P}$, some $v \geq 1$ and some $0 \leq i \leq 2^{v}$ with

$$
h^{v, *}(t) \equiv \eta>\mathcal{M} \quad t \in\left[t^{i}, t^{i+1}\right)
$$

By choosing $\left[t_{0}, t_{f}\right]$ large enough, we can assume that $i \geq 1$. Now consider the interval $\left[t^{i-1}, t^{i}\right]$, and the quantity $\sup _{t \in\left[t^{i-1}, t^{i}\right]} \Psi_{p}\left(t, h^{v, *}\right)$. By possibly modifying the value of the function $\Psi\left(\cdot, h^{v, *}\right)$ at one point, we can obtain $t^{*} \in\left[t^{i-1}, t^{i}\right]$ such that

$$
\Psi_{p}\left(t^{*}, h^{v, *}\right)=\sup _{t \in\left[t^{i-1}, t^{i}\right]} \Psi_{p}\left(t, h^{v, *}\right)
$$

Now let $\tau_{p}\left(t, h^{v, *}\right)=t+D_{p}\left(t, h^{v, *}\right)$ be the path arrival time function. According to FIFO and assumption A2, we deduce that for any $t \in\left[t^{i}, t^{i+1}\right]$,

$$
\tau_{p}\left(t, h^{v, *}\right)-\tau_{p}\left(t^{*}, h^{v, *}\right) \geq \frac{\left(t-t^{i}\right) \eta}{M^{\max }}
$$

this, together with 5.36 implies that

$$
\psi_{i j}\left(\tau_{p}\left(t, h^{v, *}\right)\right)-\psi_{i j}\left(\tau_{p}\left(t^{*}, h^{v, *}\right)\right) \geq \psi_{\min }^{\prime}\left(\tau_{p}\left(t, h^{v, *}\right)-\tau_{p}\left(t^{*}, h^{v, *}\right)\right) \geq \psi_{\min }^{\prime} \cdot \frac{\left(t-t^{i}\right) \eta}{M^{\max }}
$$


ARTICLE LINK: http://www.sciencedirect.com/science/article/pii/S0191261513000209

\section{PLEASE CITE THIS ARTICLE AS}

Han, K., Friesz, T.L., Yao, T., 2013. Existence of simultaneous route and departure choice dynamic user equilibrium. Transportation Research Part B 53, 17-30.

Inequality (5.43) implies

$$
\Psi_{p}\left(t, h^{v, *}\right)-\Psi_{p}\left(t^{*}, h^{v, *}\right) \geq \frac{\psi_{\min }^{\prime} \eta}{M^{\max }}\left(t-t^{i}\right)-\phi_{\max }^{\prime}\left(t-t^{*}\right) \quad \forall t \in\left[t^{i}, t^{i+1}\right]
$$

Integrating (5.44) with respect to $t$ over interval $\left[t^{i}, t^{i+1}\right]$ and a simple calculation yield

$$
\begin{aligned}
\int_{t^{i}}^{t^{i+1}} \Psi_{p}\left(t, h^{v, *}\right) d t-\left(t^{i+1}-t^{i}\right) \Psi_{p}\left(t^{*}, h^{v, *}\right) & \\
& \geq \frac{\left(t^{i+1}-t^{i}\right)^{2}}{2} \cdot \frac{\psi_{\min }^{\prime} \eta}{M^{\max }}+\left(t^{i+1}-t^{i}\right) \phi_{\max }^{\prime} \cdot\left(t^{*}-\frac{t^{i}+t^{i+1}}{2}\right)
\end{aligned}
$$

Since $t^{*} \in\left[t^{i-1}, t^{i}\right]$, we have $t^{*}-\left(t^{i}+t^{i+1}\right) / 2 \geq-3 / 2\left(t^{i+1}-t^{i}\right)$. Inequality (5.45) then implies

$$
\int_{t^{i}}^{t^{i+1}} \Psi_{p}\left(t, h^{v, *}\right) d t-\left(t^{i+1}-t^{i}\right) \Psi_{p}\left(t^{*}, h^{v, *}\right) \geq \frac{\left(t^{i+1}-t^{i}\right)^{2}}{2}\left(\frac{\psi_{\min }^{\prime} \eta}{M^{\max }}-3 \phi_{\max }^{\prime}\right)>0
$$

This yields the following contradiction to (5.40) and hence (5.39)

$$
\int_{t^{i}}^{t^{i+1}} \Psi_{p}\left(t, h^{v, *}\right) d t>\int_{t^{i-1}}^{t^{i}} \Psi_{p}\left(t, h^{v, *}\right) d t
$$

Part 4. By previous steps, we have obtained a uniformly bounded sequence of vector-valued functions $\left\{h^{n, *}\right\}_{n \geq 1}$ satisfying [5.39]. By taking a subsequence if necessary, we can assume the weak convergence in the Hilbert space $\left(L^{2}\left[t_{0}, t_{f}\right]\right)^{|\mathcal{P}|}$ :

$$
h^{n, *} \longrightarrow h^{*} \quad n \rightarrow \infty
$$

for some $h^{*} \in \Lambda$. We claim that for all $h \in \Lambda$,

$$
\left\langle\Psi\left(\cdot, h^{*}\right), h(\cdot)-h^{*}(\cdot)\right\rangle \geq 0
$$

Indeed, given any $h \in \Lambda$, there exists piecewise-constant approximation $\left\{h_{n} \in \Lambda_{n}\right\}_{n \geq 1}$, which converges to $h$ both point-wise and in the $\|\cdot\|_{\mathcal{L}^{2}}$ norm. According to 5.39 , we have

$$
\left\langle\Psi_{p}\left(\cdot, h^{n, *}\right), h_{n}(\cdot)-h^{n, *}(\cdot)\right\rangle \geq 0
$$

Notice that the map $h \mapsto \Psi_{p}(\cdot, h)$ is continuous with respect to the $\|\cdot\|_{\mathcal{L}^{2}}$ norm, by the continuity of the inner product, we pass 5.47) to the limit

$$
\left\langle\Psi_{p}\left(\cdot, h^{*}\right), h(\cdot)-h^{*}(\cdot)\right\rangle \geq 0
$$

The next theorem establishes the existence of SRDC-DUE with generalized Vickrey model, which is an immediate consequence of Theorem 5.3 and Theorem 5.6

Theorem 5.7. Let assumption $\mathbf{A} 1$ hold, then there exists a dynamic user equilibrium as in Definition 3.1 with an embedded network loading procedure which employs the generalized Vickrey model. 
ARTICLE LINK: http://www.sciencedirect.com/science/article/pii/S0191261513000209

$$
\text { PLEASE CITE THIS ARTICLE AS }
$$

Han, K., Friesz, T.L., Yao, T., 2013. Existence of simultaneous route and departure choice dynamic user equilibrium. Transportation Research Part B 53, 17-30.

Remark 5.8. Notice that assumption $\mathbf{A 2}$ is automatically satisfied by the Vickrey model and hence the GVM. Thus $\mathbf{A 2}$ is omitted from the statement of Theorem 5.7

As our final result, we present an important characterization of the DUE solution $h^{*}$. That is, $h_{p}^{*}(\cdot), p \in \mathcal{P}$ are uniformly bounded by some constant. One should distinguish this statement from the assumption that the path flows are a priori bounded, which is made in the proof of existence (Zhu and Marcotte, 2000). Such an informative result leads to further insights of network congestion games, and can be easily derived using analysis similar to Part $\mathbf{3}$ in the proof of Theorem 5.6

Proposition 5.9. Let $\mathbf{A 1}$ and $\mathbf{A 2}$ hold. Given a DUE solution $h^{*}(\cdot) \in \Lambda \subset\left(\mathcal{L}^{2}\left[t_{0}, t_{f}\right]\right)^{|\mathcal{P}|}$ in the sense of Definition 3.1 the following inequality must hold.

$$
h_{p}^{*}(t) \leq \frac{\phi_{\max }^{\prime}}{\psi_{\min }^{\prime}} \cdot M^{\max } \quad \forall t \in\left[t_{0}, t_{f}\right], \quad \forall p \in \mathcal{P}
$$

where constants $\phi_{\max }^{\prime}, \psi_{\min }^{\prime}$ and $M^{\max }$ are defined in (5.35), (5.36) and 5.37).

Proof. Fix an origin-destination pair $(i, j) \in \mathcal{W}$. Consider any path $p \in \mathcal{P}_{i j}$, and any two departure times $t_{0} \leq t_{1}<t_{2} \leq t_{f}$ such that $h_{p}^{*}\left(t_{1}\right)>0, h_{p}^{*}\left(t_{2}\right)>0$. Call $\tau_{1} \leq \tau_{2}$ the corresponding arrival times after traversing path $p$. By definition, the travel cost for these two drivers must be the same. Recalling (5.31) and 5.32, we have

$$
\phi_{i j}\left(t_{1}\right)+\psi_{i j}\left(\tau_{1}\right)=\phi_{i j}\left(t_{2}\right)+\psi_{i j}\left(\tau_{2}\right)
$$

By the first-in-first-out principle and assumption $\mathbf{A 2}$,

$$
\tau_{2}-\tau_{1} \geq \frac{1}{M^{\max }} \int_{t_{1}}^{t_{2}} h_{p}^{*}(t) d t
$$

Taken together, (5.49) and (5.50) yield

$$
\begin{aligned}
\left(t_{2}-t_{1}\right) & \geq \frac{1}{\phi_{\max }^{\prime}}\left(\phi_{i j}\left(t_{1}\right)-\phi_{i j}\left(t_{2}\right)\right)=\frac{1}{\phi_{\max }^{\prime}}\left(\psi_{i j}\left(\tau_{2}\right)-\psi_{i j}\left(\tau_{1}\right)\right) \\
& \geq \frac{\psi_{\min }^{\prime}}{\phi_{\max }^{\prime}}\left(\tau_{2}-\tau_{1}\right) \geq \frac{\psi_{\min }^{\prime}}{\phi_{\max }^{\prime} M^{\max }} \int_{t_{1}}^{t_{2}} h_{p}^{*}(t) d t
\end{aligned}
$$

We thus have

$$
\frac{\phi_{\max }^{\prime} M^{\max }}{\psi_{\min }^{\prime}} \geq \frac{1}{t_{2}-t_{1}} \int_{t_{1}}^{t_{2}} h_{p}^{*}(t) d t
$$

Since this is true for arbitrary $t_{1}<t_{2}$, the point-wise bound (5.48) must hold.

\section{Conclusion}

We have established the existence of the continuous-time simultaneous route-and-departure choice DUE for the generalized Vickrey model (GVM), a generalization of the Vickrey model, and plausible regularity conditions that are easy to check and rather weak. It is significant that ours is the first DUE existence result without the a priori bounding of departure rates (path flows) and the most general constraint relating path flows to the trip table. In fact, our method of proof successfully overcomes two major hurdles that have stymied other researchers: 
ARTICLE LINK: http://www.sciencedirect.com/science/article/pii/S0191261513000209 PLEASE CITE THIS ARTICLE AS

Han, K., Friesz, T.L., Yao, T., 2013. Existence of simultaneous route and departure choice dynamic user equilibrium. Transportation Research Part B 53, 17-30.

1. the set of feasible flows $\Lambda$ is intrinsically non-compact in the $\mathcal{L}^{2}$-space as well as in the $\mathcal{L}^{1}$-space; and

2. a direct topological argument requires a priori bounds for the path flows, where those bounds do not arise from any behavioral argument or theory.

Theorem 5.6 is a general result that subsumes all SRDC-DUE models regardless of the arc dynamics, flow propagation and arc delay function employed, as long as the effective delay operator is continuous. As follow-up research, the continuity of other network loading models will be identified which, when combined with the general result of Theorem 5.6, leads to the existence results of DUE with different network performance models.

\section{References}

Bressan, A., Han, K., 2011. Optima and equilibria for a model of traffic flow. SIAM Journal on Mathematical Analysis 43 (5), 2384-2417.

Bressan, A., Han, K., 2012. Nash equilibria for a model of traffic flow with several groups of drivers. ESAIM: Control, Optimization and Calculus of Variations 18 (4), 969-986.

Bressan, A., Han, K., 2013. Existence of optima and equilibria for traffic flow on networks. Networks and Heterogeneous Media, forthcoming.

Browder, F.E., 1968. The fixed point theory of multi-valued mappings in topological vector spaces. Mathematische Annalen 177, 283-301.

Evans, L.C., 2010. Partial Differential Equations. $2^{\text {nd }}$ edition. American Mathematical Society, Providence, RI.

Friesz, T.L., Bernstein, D., Smith, T., Tobin, R., Wie, B., 1993. A variational inequality formulation of the dynamic network user equilibrium problem. Operations Research 41 (1), 80-91.

Friesz, T.L., Bernstein, D., Suo, Z., Tobin, R., 2001. Dynamic network user equilibrium with state-dependent time lags Networks and Spatial Economics 1 (3/4), 319-347.

Friesz, T.L., Han, K., Meimand, A., Neto, P., Yao, T., 2013. Dynamic user equilibrium based on a hydrodynamic model. Transportation Research Part B 47 (1), 102-126.

Friesz, T.L., Kim, T., Kwon, C., Rigdon, M.A., 2011. Approximate network loading and dual-time-scale dynamic user equilibrium. Transportation Research Part B 45 (1), 176-207.

Friesz, T.L., Luque, F.J., Tobin, R.L., Wie, B.W., 1989. Dynamic network traffic assignment considered as a continuous time optimal control problem. Operations Research 37 (6), 893-901.

Friesz, T.L., Mookherjee, R., 2006. Solving the dynamic network user equilibrium with state-dependent time shifts Transportation Research Part B 40 (3), 207-229.

Han, K., Friesz, T.L., Yao, T., 2013a. A partial differential equation formulation of Vickrey's bottleneck model, part I: Methodology and theoretical analysis. Transportation Research Part B, DOI: 10.1016/j.trb.2012.10.003.

Han, K., Friesz, T.L., Yao, T., 2013b. A partial differential equation formulation of Vickrey's bottleneck model, part II: Computation and application. Transportation Research Part B, DOI: 10.1016/j.trb.2012.10.004.

Han, K., Piccoli, B., Friesz, T.L., Yao, T., 2012. A continuous-time link-based kinematic wave model for dynamic traffic networks. arXiv:1208.5141 1 .

Han, L., Ukkusuri, S., Doan, K., 2011. Complementarity formulation for the cell transmission model based dynamic user equilibrium with departure time choice, elastic demand and user heterogeneity. Transportation Research Part B 45 (10), 1749-1767.

Lighthill, M., Whitham, G., 1955. On kinematic waves. II. A theory of traffic flow on long crowded roads. Proceedings of the Royal Society of London: Series A 229 (1178), 317- 345.

Merchant, D.K., Nemhauser, G.L., 1978a. A model and an algorithm for the dynamic traffic assignment problem. Transportation Science 12 (3), 183-199.

Merchant, D.K., Nemhauser, G.L., 1978b. Optimality conditions for a dynamic traffic assignment model. Transportation Science 12 (3), 200-207.

Mounce, R., 2006. Convergence in a continuous dynamic queuing model for traffic networks. Transportation Research Part B 40 (9), 779-791.

Pang, J., Han, L., Ramadurai, G., Ukkusuri, S., 2011. A continuous-time linear complementarity system for dynamic user equilibria in single bottleneck traffic flows. Mathematical Programming, Series A 133 (1-2), 437-460.

Ran, B., Hall, R.W., Boyce, D.E., (1996). A link-based variational inequality model for dynamic departure time/route choice. Transportation Research Part B 30 (1), 31-46.

Richards, P.I., 1956. Shockwaves on the highway. Operations Research 4 (1), $42-51$. 
ARTICLE LINK: http://www.sciencedirect.com/science/article/pii/S0191261513000209

\section{PLEASE CITE THIS ARTICLE AS}

Han, K., Friesz, T.L., Yao, T., 2013. Existence of simultaneous route and departure choice dynamic user equilibrium. Transportation Research Part B 53, 17-30.

Royden, H.L., Fitzpatrick, P., 1988. Real Analysis (Vol. 3). Englewood Cliffs, NJ:: Prentice Hall.

Smith, M.J., Wisten, M.B., 1994. Lyapunov methods for dynamic equilibrium traffic assignment. In Proceedings of the Second Meeting of the EURO Working Group on Urban Traffic and Transportations, 223-245, INRETS, Paris.

Smith, M.J., Wisten, M.B., 1995. A continuous day-to-day traffic assignment model and the existence of a continuous dynamic user equilibrium. Annals of Operations Research 60 (1), 59-79.

Vickrey, W.S., 1969. Congestion theory and transport investment. The American Economic Review 59 (2), 251-261.

Wie, B.W., Tobin, R.L., Carey, M., 2002. The existence, uniqueness and computation of an arc-based dynamic network user equilibrium formulation. Transportation Research Part B 36 (10), 897-918.

Yao, T., Friesz, T.L., Wei, M.M., Yin, Y., 2010. Congestion derivatives for a traffic bottleneck. Transportation Research Part B 44 (10), 1149-1165.

Zhu, D.L., Marcotte, P., 2000. On the existence of solutions to the dynamic user equilibrium problem. Transportation Science 34 (4), 402-414. 